\title{
Myoelectric Teleoperation of a Complex Robotic Hand
}

\author{
Kristin A. Farry, Member, IEEE, Ian D. Walker, Member, IEEE, and Richard G. Baraniuk, Member, IEEE
}

\begin{abstract}
Teleoperation continues to be a primary control mode in robotics applications, particularly for robots with complex hands. This paper details a novel method of teleoperation of complex anthropomorphic robotic hands: converting the myoelectric signal (generated by the operator's muscles during movement) into robot commands replicating the motion. Myoelectric prosthetic hands have used this user interface for over two decades; however, the feasibility of using this approach for commanding more than one degree-of-freedom, as in the pincher type grip in current myoelectric hands, has been in question. The research described in this paper addresses myoelectric control of NASA/Johnson Space Center's sixteen degree-of-freedom Utah/MIT Dextrous Hand for two grasping (key and chuck) options and three thumb motions (abduction, extension, and fiexion). We discuss myoelectric signal processing approaches, data collection apparatus, and a realtime teleoperation implementation. We also present results in realtime discrimination of key and chuck grasps and offline discrimination of thumb motions. Our results include a $90 \%$ correct grasp selection rate and an $87 \%$ correct thumb motion selection, both using the myoelectric spectrum.
\end{abstract}

\section{INTRODUCTION}

$\mathbf{N}$ ASA identified improvements in control of remote dextrous manipulation devices, or teleoperation, as critical to successful maintenance of long-lived orbiting hardware such as the Space Station [1]. Required teleoperation improvements include making the user interface more intuitive (requiring less operator training) and less fatiguing (enabling an operator to work longer shifts). As teleoperated devices increase in complexity toward that of the human hand, the three degree-offreedom (DOF) joysticks now used to control the Canadianbuilt Space Shuttle Remote Manipulator System (RMS) are inadequate for intuitive and nonfatiguing teleoperation. NASA is exploring alternative hand controllers for complex robotic hands such as the Utah/MIT Dextrous Hand (UMDH) [2]; options include replica-type controllers and exoskeletons that

Manuscript received February 17, 1995; revised September 23, 1995. This work was supported primarily under a NSF Graduate Fellowship and a Zonta International Amelia Earhart Fellowship. It was also supported in part by NASA Grants NAG 9-740 and 9-845, National Science Foundation Grants MSS-9024391 and MIP-9457438, and Texas Advanced Technology Program Grant TX-ATP 003604-002. The work is continuing under a National Research Council (NRC) Research Associateship at NASA/JSC. This paper was presented in part at the 1993 IEEE International Conference on Robotics and Automation, Atlanta, GA, May 3-5, 1993. This paper was recommended for publication by Associate Editor B. Hannaford upon evaluation of reviewers' comments.

K. A. Farry is with the Engineering Directorate/Automation, Robotics, and Simulation Division, NASA/Johnson Space Center, Houston, TX 77058 USA.

I. D. Walker and R. G. Baraniuk are with the Department of Electrical and Computer Engineering, Rice University, Houston, TX 77251 USA

Publisher Item Identifier S 1042-296X(96)05332-3 fit over the operator's own hand, mapping its position directly to the robot's position.

Shortcomings of these hand controllers include inaccuracy, bulk, and weight as well as interference with some of the motions that the operator would like to command. Furthermore, none offer a way of commanding force in addition to position. Consequently, NASA/Johnson Space Center (JSC) is interested in the feasibility of using myoelectric signals to teleoperate an anthropomorphic hand, introduced in [3] and [4]. The idea of low-profile skin-surface electrodes placed on an operator's forearm which do not restrict hand motion is quite appealing. Thus, the first myoelectric teleoperation scenario (addressed in this paper) involves determining the operator's hand motion using only myoelectric signals measured from the operator's forearm. Another, somewhat less ambitious scenario (not addressed here) is using myoelectric signals to detect force exerted by an operator's hand on an active position-measuring exoskeleton.

Hand prostheses users would like additional DOF's in their prostheses, which now offer only a single-DOF pincher-type grasping [5]. However, many multiple-DOF prosthetic hand designs such as the Swedish Hand [6] have foundered on control issues [7]. These problems parallel those faced by the teleoperation community: the user interface must be intuitive and nonfatiguing to enable practical long-term use of the device. In fact, the problem faced by the amputee is larger than that faced by a teleoperator: a teleoperator can focus entirely on controlling a remote device for lengthy periods, but an amputee cannot be productive if a large portion of his or her energy and concentration is spent controlling an artificial hand.

An extensive review of the myoelectric control literature indicates that either the space teleoperation or prosthesis improvement scenario requires advances in the state of the art of myoelectric control. NASA and a group of medical and prosthetics specialists, prosthesis users, and insurance industry representatives collaborated in a 1991 workshop to identify research objectives in prosthetic hands [8]. The mediumterm objectives of this coalition include improvements in myoelectric control to improve hand positioning and grasping. These objectives guide our research.

We are exploring myoelectric teleoperation by a fullylimbed subject, as we believe that this defines an "upper bound" on the performance of myoelectric control with limbdeficient prosthesis users. We are emphasizing myoelectric signal processing techniques that will result in "intuitive" control of multi-fingered hands on the order of complexity of the UMDH. This paper outlines our approach in determining 


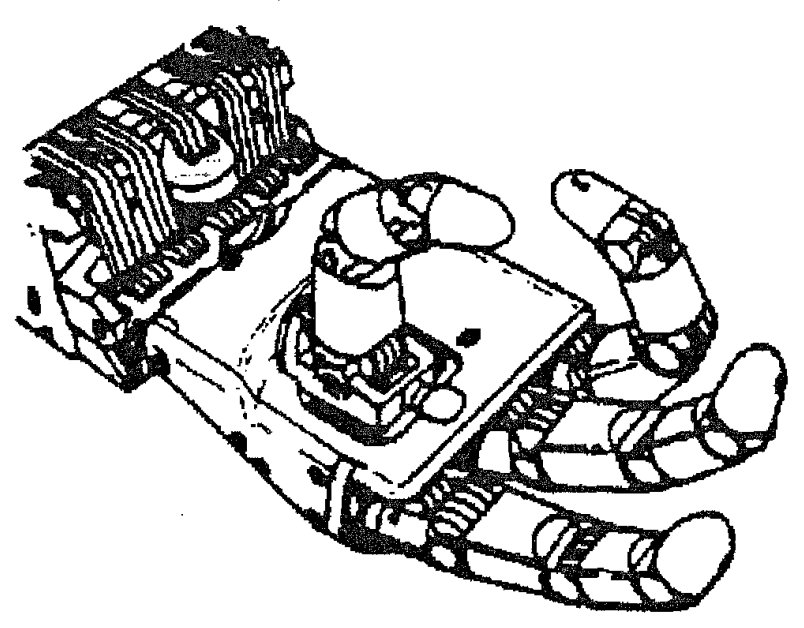

(a)

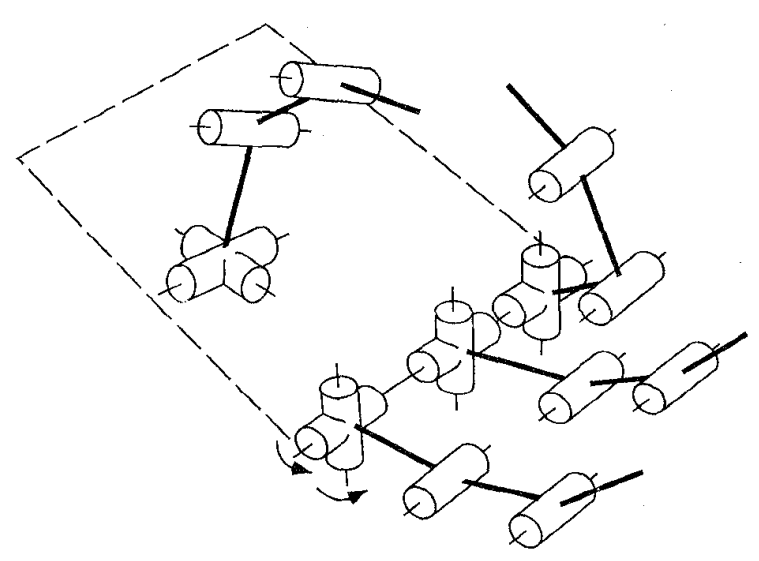

(b)

Fig. 1. The Utab/MIT Dextrous Hand (UMDH) and its kinematics.

the extent of myoelectric control possible with fully-limbed subjects. It covers a brief summary of the myoelectric signal processing state of the art and how it has influenced our choice of research directions, our experimental setup at NASA/JSC, and results.

\section{RELATED PREVIOUS WORK}

\section{A. Robotic Hands and Grasping}

The theory of grasping and manipulation by multifingered robot hands has been the subject of much research in the past decade. (See, for example, the books by Mason and Salisbury [9] and Cutkosky [10].) Researchers have proposed a number of sophisticated algorithms for grasp synthesis, mechanics, and control. Some robot hands have been constructed and tested, notably the UMDH [2] and the Salisbury Hand [11]. The anthropomorphic nature of the 16-DOF UMDH (Fig. 1) made it a logical choice for our myoelectric teleoperation study. Jacobsen and his colleagues at University of Utah designed the UMDH [2]; Hollerbach and Narasimhan at MIT [12] developed its kinematics and some control.

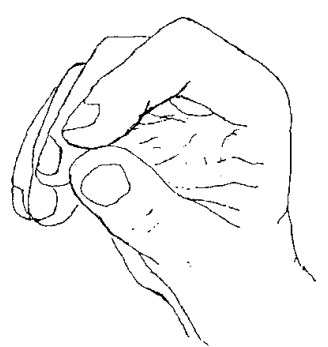

(a)

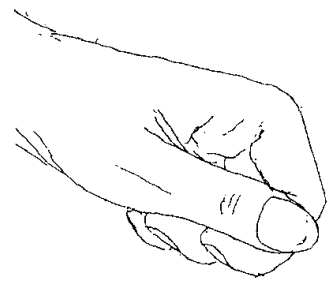

(b)
Fig. 2. (a) Chuck and (b) key grasp primitives.

The UMDH has three 4-DOF fingers which have three flexion joints each, plus an abduction joint perpendicular to the first flexion joint. It also has a 4-DOF thumb which has three flexion joints and an opposition joint intersecting the first (base) flexion joint. These two intersecting joints at the thumb's base allow an excellent approximation of the opposition capability of the human hand. Duplicating thumb abduction and adduction with this particular two-axis choice for the base of the thumb requires some mapping, but does not limit the UMDH in performing motions of the human hand.

The UMDH is a complex testbed for evaluating grasping strategies. Currently, however, the experimental state of the art lags the theory. Many proposed algorithms are too complex or require sophisticated sensors now unavailable. Speeter suggested and validated one promising approach [13]. He uses a small set of basic grasp primitives, each of which is simple to program. This approach seems well-suited for application to teleoperation using a probably small set of myoelectric signals.

In this work, we seek to identify a set of basic motion primitives and distinguish between them in the myoelectric signal. We then adapt Speeter's concept to teleoperate the robot hand.

\section{B. Human Grasping and Manipulation}

Working prior to the robotic hand research efforts, the prosthetics community reached a rough consensus that there are five types of grasp important in a person's daily activities: 1) three-jaw chuck or pincher grasp used to hold small objects [Fig. 2(a)]; 2) lateral grasp, most often called a key grasp because it is used to hold a key while unlocking a door [Fig. 2(b)]; 3) hook grasp, used to carry items such as books or a briefcase; 4) spherical grasp, where the thumb and fingers are wrapped around a spherical object; and 5) cylindrical grasp, where thumb and fingers are wrapped around a cylindrical object [14]-[16]. Some consider the spherical and cylindrical grasps to be variations of the ability to make a fist. Others argue that the flattened hand (with thumb rotated completely out of opposition of the fingers) is also a grasp of sorts, as it is essential in supporting flat objects such as trays. The relative importance of these grasps is a function of a person's occupational and personal needs.

Human hand control occurs at another level besides grasping: independent finger and thumb control [17]. We use individual thumb and finger control to manipulate small objects. 


\section{Prosthetic Hands}

Commercially available electric hand prostheses are capable of the chuck grasp, with the capability to open the fingers in a two-jaw pincher fashion to give some cylindrical grasping capability [5]. More advanced prostheses such as [18]-[21] have incorporated chuck and key grips, with spherical and cylindrical grasp options being provided by passive finger compliance. Some designs incorporated enough thumb control to change grasps from chuck to key and back. Only a few have had individual finger control.

Weight, size, cost, and reliability of the added functionality of these advanced prostheses were major reasons why they never became commercial products; however, recent advances in miniaturizing hardware and lowering power consumption and costs suggest that these problems may now be secondary to the control and user interface problems. In fact, the longest (over 15 years) multifunction hand project, the Swedish hand, ended with this conclusion [6]. Hence, our work focuses on improving the myoelectric user interface. In this paper in particular, we explore new ways to myoelectrically command grasping and thumb control primitives.

\section{Myoelectric Control}

We encourage the reader to study [22]-[24] for detailed physiological and mathematical descriptions of the development of the myoelectric signal during muscle contraction. Briefly, an electrode placed on the skin surface can measure the passing of the action potentials propagating along muscle fibers underneath the electrode as the fibers contract. Skeletal muscle fibers are innervated (and hence stimulated to contract) in small groups known as single motor units (SMU's), the smallest voluntary contractible group. A single action potential in an SMU is associated with a twitch; sustained contractions require repeated firings (resulting in a string of action potentials) of an SMU. As more force is needed, more SMU's are fired or recruited. The electrode records the sum of all these action potentials after they propagate through the tissue to the skin surface.

Research in using the resulting myoelectric signal (also called the electromyographic or EMG signal) to control environments or prostheses dates from the late 1940s [25], [26]. By the early 1970s, researchers were treating the myoelectric signal as an amplitude modulated signal whose amplitude was roughly related to the force developed in the muscle generating it. The consensus was that most of the information in a myoelectric signal is in the amplitude [22]. In the late 1970s, the model matured to treat the myoelectric signal as amplitude modulated Gaussian noise whose variance is related to the force developed by the muscle [24], [27].

Consequently, most commercial myoprocessors used in prosthesis control are now based on only one dimension of the myoelectric signal, the variance or mean absolute value (which is directly related to the variance). A few systems use the rate of change of the myoelectric signal's variance. Researchers have successfully refined variance estimation from the myoelectric signal [24], [27]--[32]. Parker's work forms the basis of control of multiple functions using different variance levels on a single channel [24]. Hogan's work was particularly significant in eliminating low frequency noise from the variance estimates due to the spatio-temporal sampling artifact inevitable with skin surface electrodes [22], [31]. Jacobsen [32] and Meek [33] refined use of the rate of change of mean absolute value in elbow control of the Utah arm. A version of the Swedish Hand used rate of change of mean absolute value to switch control functions [19]. We investigated these variance estimation results in operating a proportionally controlled grasp force with a three-fingered robotic hand.

These variance estimation techniques require a separable (i.e., different) muscle contraction for each function commanded, making simultaneous control of two or more joints very difficult. A number of researchers, beginning with Wirta and Taylor [34], examined linear combinations of myoelectric mean absolute value estimates from multiple channels to select different functions. The Swedish Hand developers applied these methods to selecting wrist motion and grasp [6], [35], [36]. The Japanese research team applied the technique to wrist control in the Waseda Hand 3 [18]. Jacobsen [37] and Jerard [38] extended and formalized the mathematics for this approach and applied it to upper limb above-elbow prostheses. These variance-estimating approaches require at least one electrode pair and signal processing channel for each muscle used, up to a dozen in some above-elbow experiments. Furthermore, variance estimating myoprocessors can be used only on superficial muscles [31], while most motions involve both superficial and deep muscles. In fact, any deep muscle activity reaching a variance estimating myoprocessor is erroneously interpreted as superficial muscle activity.

Thus, previous simultaneous control multi-function systems based on the variance-estimating philosophy required tedious tailoring to each individual and did not automatically accommodate day-to-day variations in individuals. Even after extensive training, clinical tests of multifunction prostheses based on this approach showed that amputees must concentrate on the contractions to consistently reproduce desired myoelectric patterns [6]. The effort involved has resulted in amputee rejection. It appears that we must exploit all information in the myoelectric signal, not just the variance estimate or mean absolute value, in order to obtain multifunction sensitivity that is intuitively easy to use.

Some researchers have considered shape and spectral characteristics of the myoelectric signal in addition to variance. Recent findings suggest that there is information in the myoelectric spectra, if we can understand its coding. Examples include:

1) Small muscles controlling small fine motion generally have fewer fibers per SMU than large coarse-motion muscles. The brain recruits the small muscles' full SMU complement earlier in the force profile and varies their firing rate across a wider range to modulate force output after recruitment. Consequently, they have power spectra containing more high-frequency activity than larger muscles [39]. 
2) Tissue (including other muscles) between the active muscle and the measuring electrode acts as a low pass filter to myoelectric signals, thus excessive lowfrequency power densities may indicate cross-talk from adjacent muscles [40].

3) Action potential conduction velocity decreases with fatigue, causing gradual shifts in power to lower frequencies during sustained forceful contractions [40], [41].

4) SMU recruitment order (i.e., the order in which individual SMU's are initially fired) is stable for a given task [42]. Short-time spectra of myoelectric signals associated with a given rapid movement do not vary as much as previously thought [43], [44].

The full spectrum of the myoelectric signal has been examined using techniques involving statistical patterns and spectral analyzes [45]-[50]. Lindstrom and Magnusson [40], DeLuca [42], Hannaford and Lehman [43], and Farry [44] report evidence of movements having distinct spectral signatures. Hudgins recently used spectrum-related features in the initial muscular recruiting phase of arm motions to select up to six functions of an upper limb prosthesis from a single myoelectric signal [51].

\section{APPROACH}

\section{A. Theory}

Hudgins' [51] use of spectrum-related parameters such as zero crossing and slope changes in short windows and Hannaford's [43] analysis with spectrograms led us to focus on the time-varying spectrum of the myoelectric signal in our myoelectric teleoperation research. We are studying the correlation between the myoelectric spectrum in the initial recruiting phase of a motion and the type of motion. We are following the lead of Saridis [48], Doerschuk [49], Kelly [50], and Hudgins [51] in using multiple-muscle myoelectric signals instead of the traditional single-muscle signals. However, our work differs from the previous spectrum-based work of other researchers [46], [49], [50] in that we are using a nonmodelbased frequency spectrum estimate to discriminate different grasping and thumb motions. Also, previous work focused on arm, not hand, motions.

First, we consider Hudgins' scheme [51]. This approach uses mean absolute value, mean absolute value slope, zero crossings, and waveform length in six nonoverlapping segments, each $40 \mathrm{~ms}$ long, covering the initiation phase of the motion. A multilayer perceptron neural network with back propagation uses these features to classify the myoelectric signatures into the motions generating them. Hudgins reported $69-98 \%$ accuracy (depending on the subject) in classifying a set of six arm motions.

We next consider a hybrid between Hudgins' scheme and Hannaford's analysis [43], an approach that divides the initiation phase of the signal into six $40 \mathrm{~ms}$ windows but, instead of Hudgins' implicit time-frequency parameters, computes the window's spectrum in each segment. In our first spectrumbased approach, we apply a Hamming window [52], w[n], to each myoelectric signal segment $x[n]$. We then compute the

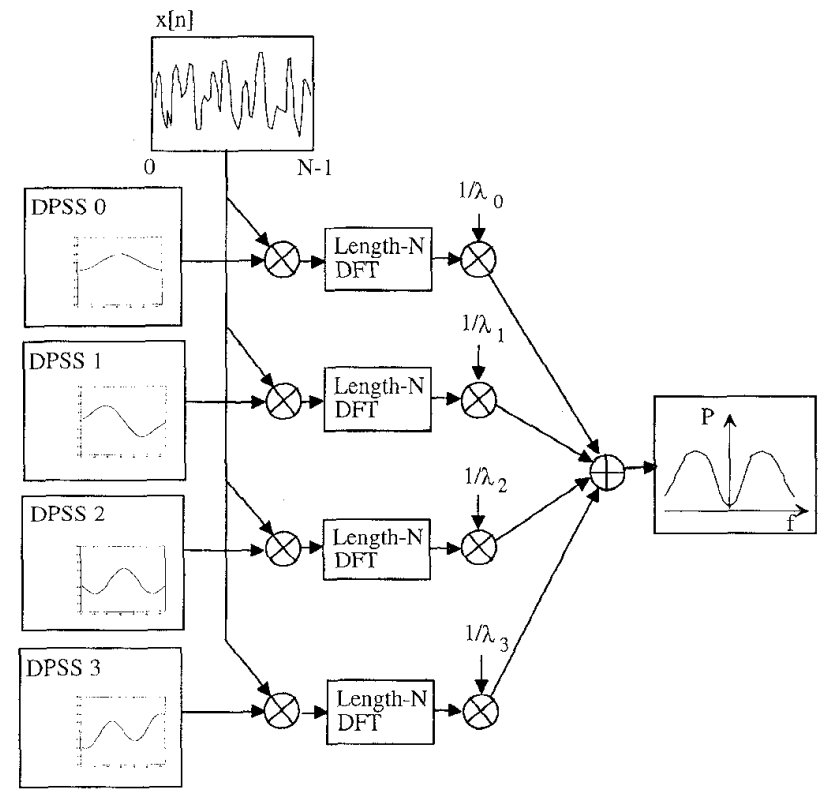

Fig. 3. Thomson's multiple window method, crude version, for $N W=2$. Note the four length- $N$ discrete prolate spheroidal sequences (DPSS's) used as data windows. We multiply our data stream $x[n]$ by each window and then compute its eigenspectrum using the discrete Fourier transform (DFT). Next, we weight each eigenspectrum with the eigenvalue associated with its DPSS $\left(\lambda_{k}\right)$ and sum all the weighted eigenspectra to get the Thomson's spectrum of $x[n]$.

periodogram [53]

$$
P(f)=\frac{1}{N}\left|\sum_{n=0}^{N-1} x[n] w[n] e^{-j 2 \pi f n}\right|^{2}
$$

using the FFT at points

$$
f=\frac{2 \pi k}{N}, \quad k=0,1, \cdots, N-1
$$

in each of the six windows. The result is a spectrogram, albeit a sparse one. Our $w[n]$ choice (the Hamming window) reduces bias associated with using only a rectangular window on this infinite signal. The Hamming window does reduce frequency resolution, however.

To use myoelectric spectral features in a realtime control system, we need a spectral estimation method that 1) works well on small windows of a single time stream or realization of the signal; 2) does not suffer from bias and variance that obscures the spectral features we are trying to detect; and 3) incorporates no incorrect assumptions about the structure of the signal. Unfortunately, the simple-to-compute spectrogram suffers from substantial bias and variance [64]. AR model-based estimators [47] have lower variance than the spectrogram, but their all-pole assumption matches certain motion artifacts in the measurement process much better than the basic physiological signal. Consequently, their output can also be badly biased [64].

Thus, we turn to a more recently developed nonparametric spectral estimator, Thomson's multiple window method (MWM) [54]. The spectrum estimation literature reports that 
Thomson's MWM gives dramatically lower bias and variance than the periodogram, without introducing a potentially misleading model [44], [54], [55]. Fig. 3 shows Thomson's simplest algorithm, which Thomson called the crude MWM [54]. Instead of applying one ad hoc window such as the Hamming window to each signal segment, we apply a set of $N$-point windows to the each segment that are 1) mutually orthogonal and 2) optimally concentrated in frequency. The particular windows - discrete prolate spheroidal sequences (DPSS's) - are the inverse discrete-time Fourier transforms of the eigenfunctions of a cascaded band-limiting (to $[-W, W]$ ) and time-limiting (to $[0, N-1]$ ) operation [56]. The crude Thomson's MWM power spectral density estimate on a length- $N$ data window is

$$
\hat{P}_{x x}(f)=\frac{1}{2 N W} \sum_{k=0}^{2 N W-1} \frac{1}{\lambda_{k}}\left|x_{k}(f)\right|^{2}
$$

where $x_{k}(f)$ are the eigenspectra

$$
\left|x_{k}(f)\right|^{2}=\left|\sum_{n=0}^{N-1} x[n] \nu_{k}[n] e^{-j 2 \pi f n}\right|^{2} .
$$

Here, $\nu_{k}[n]$ is the $k$ th DPSS window and $\lambda_{. k}$ is its corresponding eigenvalue. The DPSS windows are easy to calculate [65]. $W$ is the resolution of the estimate. We use only the largest $K=2 N W$ eigenvalues and their corresponding eigenfunctions in the estimate; $K$ controls the tradeoff between the bias and additional spectral information contributed by the higher order DPSS's. Larger $K$ (and hence large $W$ ) gives larger bias but smaller variance. Fig. 3 shows the $K=4$ case.

A detailed Thomson's MWM analysis of myoelectric signals appears in [57]. In myoelectric signal analysis, it introduces considerably less variance than the periodogram and less bias than the autoregressive model approach. The MWM is also less sensitive to window size than the periodogram.

Recall that we extend the periodogram (a stationary spectrum estimator) into the spectrogram (a time-frequency estimator) by translating the data window in time. Similarly, we extend Thomson's MWM (also a stationary estimator) into the short-time Thomson transform (STTT) by translating the DPSS's in time [58]. We can, for instance, analyze a $192 \mathrm{~ms}$ window covering the motion's initiation with three nonoverlapping $64 \mathrm{~ms}$ Thomson's spectra.

STTT analysis suggests that the myoelectric signal is more stationary than spectrogram analyzes imply [58]. Thus, using the MWM method makes the relative size and timing of each myoelectric window captured for control analysis less critical. Consequently, we introduce a new myoelectric control algorithm based on the STTT.

\section{B. Application}

1) Data Collection and Analysis Implementation: At NASA/JSC, we developed a unique myoelectric data collection system (MDCS) to capture up to eight myoelectric data streams while simultaneously recording the motion of the subject's hand and arm [44]. We also developed a myoelectric teleoperation demonstration system (MTDS) around NASA/JSC's UMDH [44].

Fig. 4 diagrams the MDCS. We use an EXOS ${ }^{\mathrm{TM}}$ exoskeleton to measure the subject's motions. The $\operatorname{EXOS}^{\mathrm{TM}}$, originally used by NASA/JSC as a master to teleoperate the UMDH, measures joint-angle related parameters for four joints on the thumb and each of three fingers (index, middle, and ring) [59]. The exoskeleton data can be used in either of two ways. First, we can use the raw voltages directly to identify a particular motion (such as a grasp), align motion trials in time, or to check for consistency between a group of similar motions. Second, we can calibrate the exoskeleton to an individual and convert its measurements to the actual joint angles or fingertip positions of the subject. Developing this conversion capability required analysis of the four- and five-bar linkages formed between the EXOS ${ }^{\text {TM }}$ and the subject's digits. We modified and extended the 1990 Wright and Stanisic [60] exoskeleton calibration work for this project [44].

Each channel of the Grass Instruments Model 12 amplifier [61] used to measure myoelectric signals consists of a differential amplifier, a high-pass filter (with roll-off frequency adjustable from $0.01-300 \mathrm{~Hz}$ ) to block DC and motion artifact, a low-pass filter (adjustable from $30-20000 \mathrm{~Hz}$ ) to limit aliasing, an adjustable-gain amplifier stage, and isolation to protect the human subject from electric shock hazards of power supply and computer equipment. This sequence amplifies the differential myoelectric signal from skin-surface electrodes (around 1 millivolt in amplitude) to several volts. Differential amplifier input somewhat reduces the $60 \mathrm{~Hz}$ interference (typically much larger than the myoelectric signal) from lights and equipment.

The Model 12 system is a clinical system originally designed for electroencephalogram (EEG) recording. It has lower input impedance $(20 \mathrm{k} \Omega$ ) than more recent laboratory systems (up to $1 \mathrm{G} \Omega$ ) for myoelectric signal measurement. To control motion artifact (i.e., spurious signals caused primarily by relative motion between the skin and the electrode), it must be used with wet, recessed electrodes and some skin preparation (removing the outer layer of dead skin cells). Skin preparation is not acceptable for an operational system, but this system is adequate and cost-effective for our research data collection.

We connected both exoskeleton and myoelectric amplifiers through Burr-Brown MPV950S A/D boards to 68020-based Ironics IV3204 and IV3201 microcomputers. These capture up to 32 channels of data at 1000 samples per second per channel. An 80386 Radix PC transfers data to MATLAB ${ }^{\text {TM }}$ format files for analysis. We use The MathWorks' MATLAB ${ }^{\mathrm{TM}}$ software, Version 4.2, for off-line data analysis and plotting [62].

2) Demonstration Implementation: The myoelectric teleoperation demonstration system (MTDS) combines the UMDH (as the robotic hand being controlled) with the Grass Instruments Model 12 amplifiers and modular signal processing and control software running in realtime. Fig. 5 gives the demonstration hardware overview.

This demonstration system uses neural networks to classify myoelectric signatures; consequently, the system incorporates a training as well as an operational mode. Two computers (the 68020 Ironics IV3204 and 80386 Radix PC used in the 


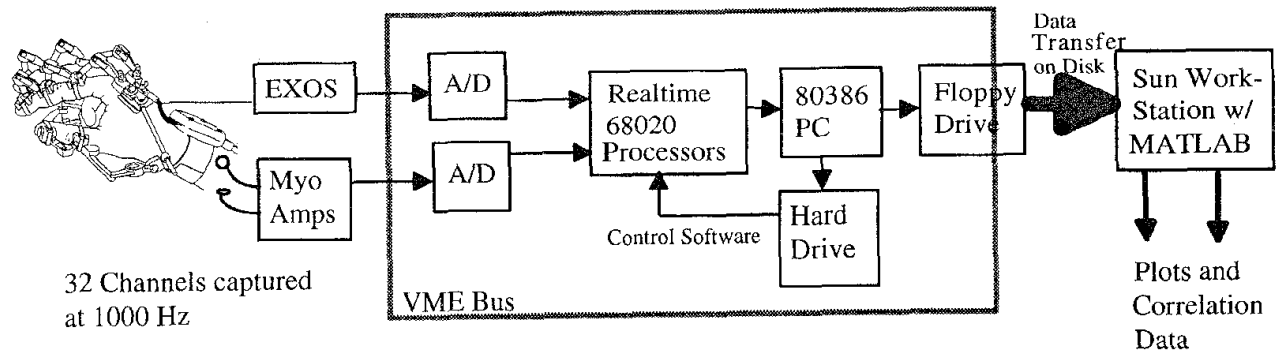

Fig. 4. Myoelectric data collection system (MDCS). Note the use of the exeoskeleton to measure hand motion data simultaneously with myoelectric measurement.

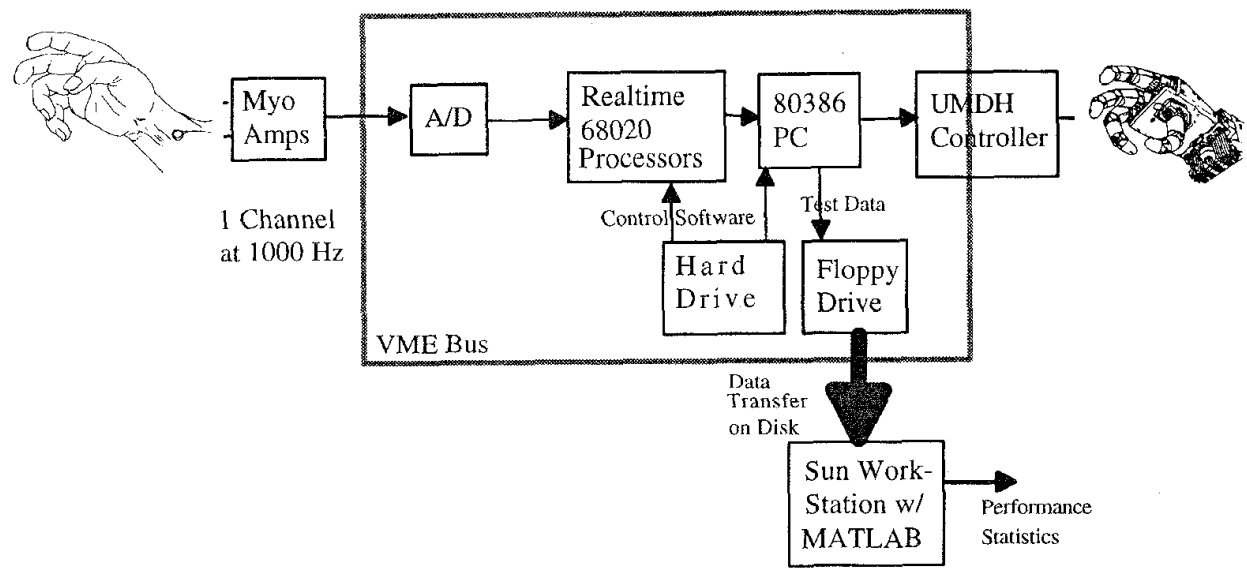

Fig. 5. Myoelectric teleoperation demonstration system (MTDS).

data collection system) run the myoelectric signal capture and processing software and interface with the UMDH controller.

Software in the VME bus-mounted 68020 Ironics IV3204 microcomputers captures myoelectric data from the Model 12 amplifiers via the Burr-Brown MPV9508 A/D. This software module temporarily buffers the myoelectric signal. It also continuously computes the energy of the last $M$ samples, then the change in energy between the current $M$-sample window and the previous one. When this change in energy between windows exceeds a threshold value, this software finishes capturing enough data past this threshold crossing for the classification algorithm. Then, it triggers a signal training data capture or classification algorithm running in the 80386 Radix PC, also mounted in the VME bus.

A Radix PC software module computes a set of features from the classification window. This window's size dependent on the classification algorithm, but including data bracketing the trigger point to insure that it includes the signal's initiation phase. Any C-implemented feature computation module can be incorporated for feature computation. We have also embedded an fast Fourier transform (FFT) for various spectral estimators. In the future, a digital signal processor (DSP) call will replace this FFT algorithm.

The software next scales the classification window features and then either saves them for neural network training (if the system is in a training mode) or presents them to a previouslytrained neural network (if the system is in an operational
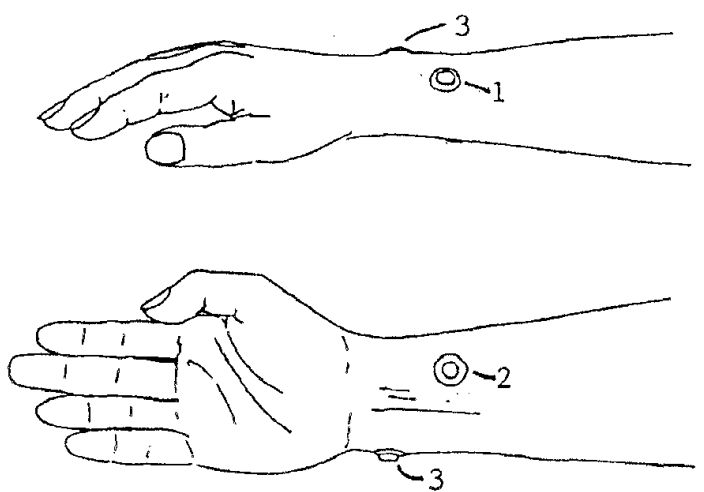

Fig. 6. Electrode locations used for the grasp demonstration (subject 1) and thumb motion discrimination (subject 2). 1) APB/EPL; 2) FPL; and 3) reference.

mode). The embedded neural network software module is the 1988 OWL Neural Network package [63]; it also runs in the Radix PC.

In the training mode, the training supervisor software associates the signature's feature set with the randomly selected grasp choice used to prompt the operator, unless the operator indicates that the current feature set is from a false trigger or unacceptable. If a false trigger, the software associates the feature set with the no grasp. If unacceptable (for example, the operator made the wrong grasp), the software discards the feature set. 
Neural network training begins only after the system collects the user-specified number of training data sets. These sets are split into training ( $75 \%$ of total) and training test ( $25 \%$ of total) groups. The software then trains the network until: 1) the user stops it; 2) the combined actual selection error on the training and training test groups (based only on the number of correct grasp selections) hits a minimum; 3) the least squared output error (LSE) for the training group goes below a user-specified value; or 4) the system meets some user-specified number of training iterations. The user can repeat the training many times with different termination criteria if desired. The user can also add more training signatures.

When the neural network is fully trained and operational, the two outputs of the neural network select one of the two grasp options (key or chuck in these trials), a no grasp option (false trigger case), or an ambiguous grasp option (legitimate trigger but unrecognizable motion). If the neural network selects one of the two grasp options, a UMDH control module sends the robot to a position defined in the corresponding grasp-macro data file. Current macros include KEY_GRASP, CHUCK_GRASP, and OPEN_GRASP. Defining a new macro consists of defining a new terminal position and storing it in a grasp-macro data file.

The UMDH holds the selected grasp position until the next time the trigger algorithm sees a threshold crossing. The system assumes that this burst corresponds to the operator opening her or his hand and commands the UMDH to OPEN_GRASP. In a free-running mode, the system simultaneously rearms itself for the next grasp. In a test-monitoring mode, the system pauses to show the corresponding myoelectric signature and store the computed features on disk for later analysis on a MATLAB $^{\text {TM }}$ workstation.

\section{RESULTS}

\section{A. Offline Analysis}

1) Grasp Discrimination: We used the MDCS to collect myoelectric signatures during 60 chuck and key grasps from a single subject. We used these signatures in an offline or nonrealtime evaluation of several discrimination approaches prior to our realtime implementation effort. Fig. 6 shows the electrode locations. We placed the electrodes where they would see the most muscle activity during thumb motion: 1) Abductor Pollicis Longus and Extensor Pollicis Brevis pair (APL/EPB) (most active is thumb extension and abduction) and 2) the Flexor Pollicis Longus (FPL) (most active in thumb flexion) [15]. Electrode 3) is a ground reference electrode.

We selected these electrode positions as a candidate configuration for our single-channel grasp discriminator because these extrinsic thumb muscles have different roles in the beginning of the chuck and key grasps. The thumb abducts and rotates away from the palm before flexion in the chuck grasp, but only flexes in the key grasp. The monopolar differential configuration (referenced to a ground electrode over electrically inactive tissue near the styloid process of the ulna) results in signals containing all muscle activity within approximately a centimeter of each electrode.
The two most significant grasp discrimination offline trials were 1) a duplication of Hudgins' University of New Brunswick (UNB) scheme for arm motion discrimination [51] and 2) the spectrogram scheme described above (1), which eventually became our first realtime implementation on the MTDS [44].

The first of these, the UNB scheme, was our performance baseline. Recall that Hudgins reported 69-98\% accuracy (depending on the subject) in classifying a set of six arm motions. Our application of this approach to grasp data yielded $80 \%$ correct classification of two grasps (chuck and key) in the best case.

Our spectrogram approach achieved a 93\% correct classification rate on the same data set on which the UNB scheme scored $80 \%$. Fig. 7 shows its realtime application to a single grasp's myoelectric signature. While use of the Hamming window reduces spectral resolution somewhat from that of the unwindowed spectrogram, our current hardware restricted realtime resolution further. We computed four spectral magnitude averages covering the $75-250 \mathrm{~Hz}$ region of the spectrum in each window, for a total of 24 feature inputs to a multilayer perceptron neural network. Muscle action potential propagation factors such as conduction velocity, number of recruited SMU's, and tissue filtering shape the upper (75-250 $\mathrm{Hz}$ ) region of the myoelectric spectrum. SMU recruiting dynamics dominate the lower $(0-75 \mathrm{~Hz})$ region. We found the lower spectrum to be much less stationary than the upper. This, coupled with the spectrogram's bias and motion artifact in the lower region, led us toward use of the upper spectrum only. Among the many spectrogram variations we evaluated off-line, the upper spectrum methods performed much better than the full $(0-250 \mathrm{~Hz})$ spectrum methods.

2) Thumb Motion: We also collected between 50 and 60 myoelectric signatures for each of three simple thumb motions-abduction, extension, and flexion (Fig. 8)-from a second subject, with careful attention to limiting motion artifact and wrist motion. These simple motions are more consistent and easier to align in time (based on their exoskeleton signatures) than the grasp signatures. We compared spectral estimates of these signals from the periodogram method, autoregressive (AR) models [45], [64], and Thomson's MWM [54], both with and without prewhitening [65], [66]. Since the myoelectric spectra is nonstationary, we performed this analysis for relatively short (compared to the duration of the motion) windows (32-64 ms) with 10-64 ms spacing [44]. Fig. 9 illustrates the dramatic difference in variance between the Hamming periodogram and the MWM for 60 thumb abduction signatures. These variances include variance contributed by the spectrum estimation method as well as that from physiological and measurement artifact sources. The lower variance of the MWM signature translates directly into increased classification performance, as it makes motionspecific spectral differences more usable.

Our MWM analysis showed differences between the myoelectric spectra associated with thumb abduction, extension, and flexion. Fig. 10 superimposes mean spectral signatures for 60 abductions, 53 extensions, and 54 flexions of the thumb, recorded from the same locations used for grasp 


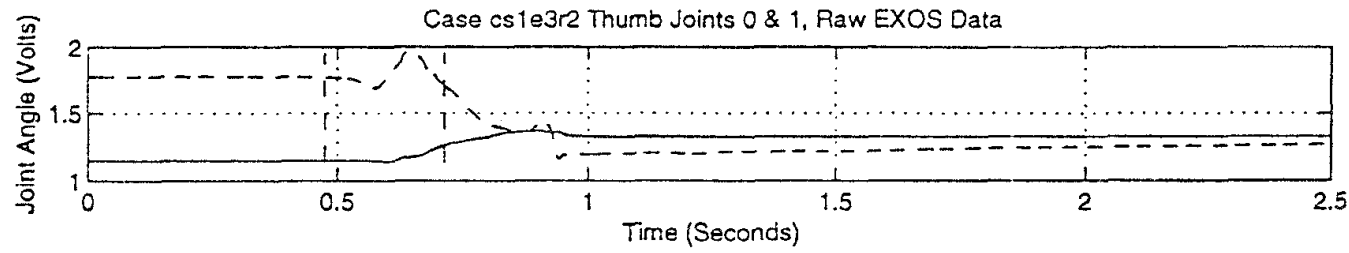

(a)

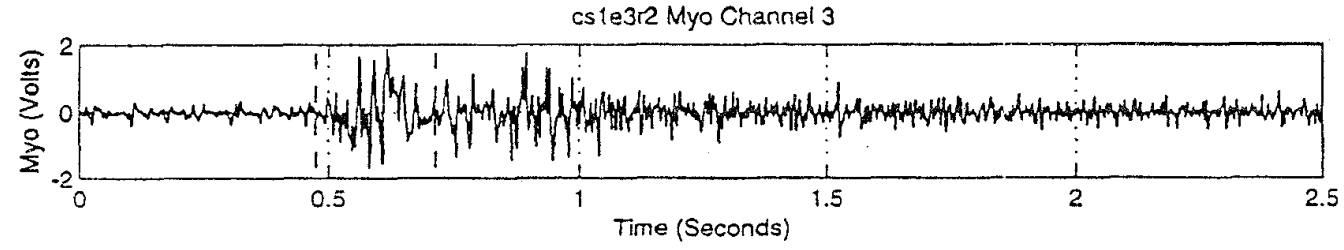

Analysis Window, 0.4750 .714 , MAV thres $=0.2$, Offset $=60$

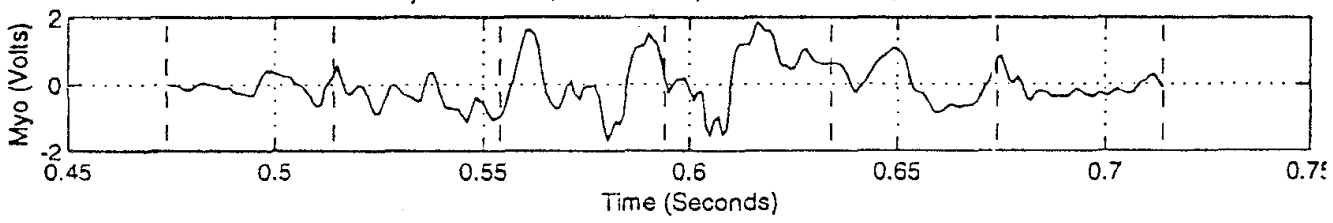

(b)
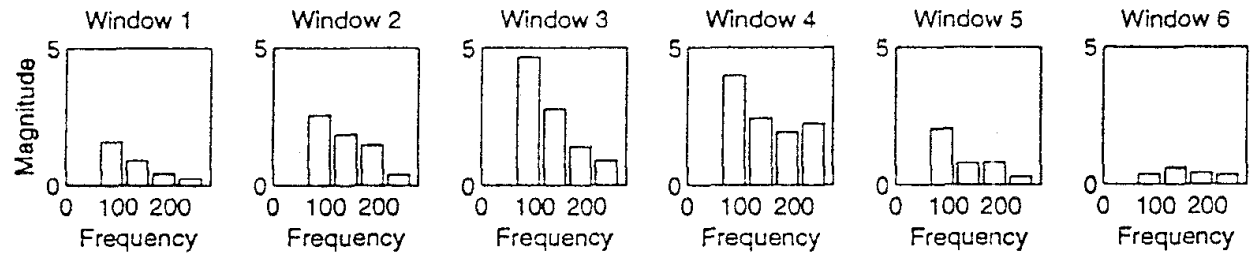

(c)

Fig. 7. Six window Hamming spectrogram features (bottom) computed from a single chuck grasp's (top) myoelectric signature (middle).

discrimination (Fig. 6). These spectral differences correlate with the underlying physiological processes. The flexion spectrum has more energy at lower frequencies. FPL is most active in thumb flexion. It is deeper that the APL/EPB pair. Thus, tissue filtering (low pass and proportional to distance traveled through the tissue) removes much higher frequency activity.

The peaking of the extension average near $60 \mathrm{~Hz}$ is probably due to greater motion artifact in that signal than in those from the other motions. The extension motion was typically faster than the abduction or flexion, and thus more prone to motion artifact. The higher average speeds for extension also account for the overall greater energy in its spectra. The abduction spectral peak is the broadest. While APL is a primary mover in thumb abduction, the motion also requires significant FPL activity in an antagonist role.

We conducted an offline comparison of the Hamming spectrogram and the short-time Thomson transform in discriminating thumb abduction, extension, and flexion [67]. In these three-way discrimination trials, using three nonoverlapping, $64 \mathrm{~ms}$ windows covering $192 \mathrm{~ms}$ at the motion's start, the STTT performance averaged approximately $86 \%$ correct classification. It outperformed the Hamming spectrogram using the same windowing scheme, which averaged approximately $75 \%$ correct classification.
Recall that muscle fatigue causes a reduction in the muscle action potential conduction velocity, which in turn shifts the spectral energy toward lower frequencies. This effect usually accompanies prolonged, high force contractions. We checked for shifts in mean frequency and power over time in our thumb data set. Since this data set involved nearly 170 recorded thumb motions and many additional unrecorded thumb motions over several hours' time, we feel that the subject's fatigue levels at the end of the data collection session might be comparable to that experienced in a realistic teleoperation scenario. We found no appreciable changes in the spectrum's mean frequency and power over time, probably because these command motions are neither prolonged nor high force.

\section{B. Realtime Implementation}

In parallel with our offline work, we addressed realtime implementation problems in using nonparametric spectral estimators in a myoelectric control system. The results of the comparative spectral analysis and off-line experiments, combined with the throughput limits of the UMDH's conventional microcomputer controller and $1000 \mathrm{~Hz}$ sample rate limitation, suggested basing the first realtime myoelectric teleoperation demonstration on the $75-250 \mathrm{~Hz}$ range of $40 \mathrm{~ms}$ Hamming- 

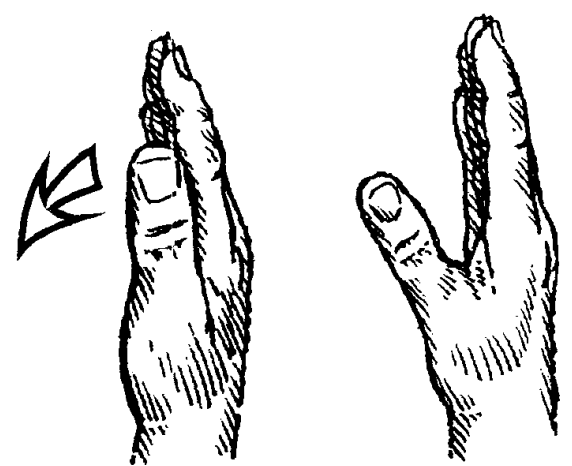

(a)
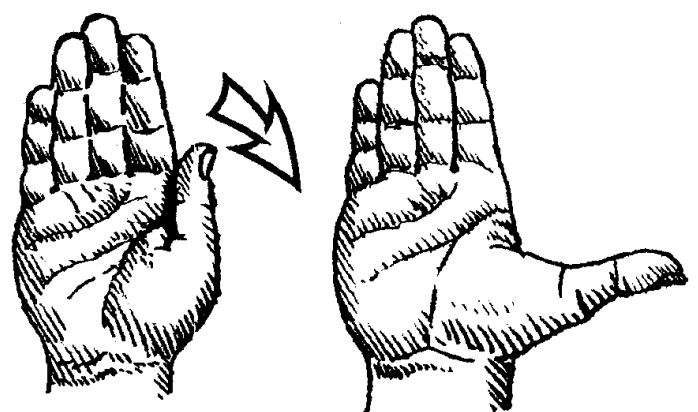

(b)
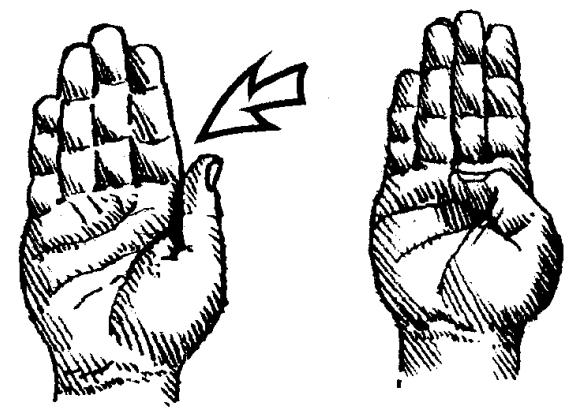

(c)

Fig. 8. We collected myoelectric signatures associated with thumb (a) abduction, (b) extension, and (c) flexion to evaluate motion discrimination algorithms.

windowed spectrograms. In these short windows, the variance problems of the spectrogram appeared tolerable. Thus, the classification features in this first myoelectric teleoperation demonstration are Hamming spectrogram magnitudes in the $78-265 \mathrm{~Hz}$ range of six consecutive $40 \mathrm{~ms}$ windows. The first of these windows begins $60 \mathrm{~ms}$ before the signal meets the trigger condition.

The system pads the $40 \mathrm{~ms}$ data windows to make them length 64 . The zero padding to the next power of two actuaily makes the FFT computationally more efficient. The system next consolidates the 12 FFT outputs in the $78-265 \mathrm{~Hz}$ range down to four by summing adjacent triplets. This gives the network a crude representation of the spectral energy distribution. Higher resolution representations require larger neural networks and slow the demonstration system to the point that the operator no longer perceives the system as "realtime."

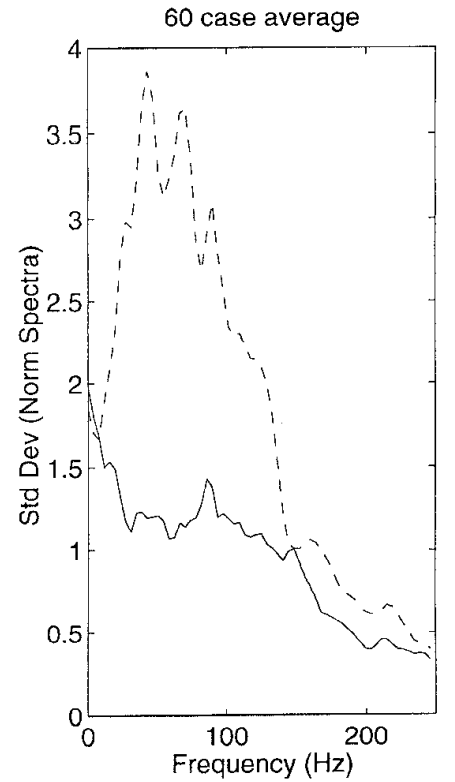

Fig. 9. Standard deviation computed across area-normalized myoelectric spectra (64 ms window at motion start) for 60 thumb abductions shows that Thomson's MWM (-) produces lower variance estimates than the Hamming periodogram (- -).

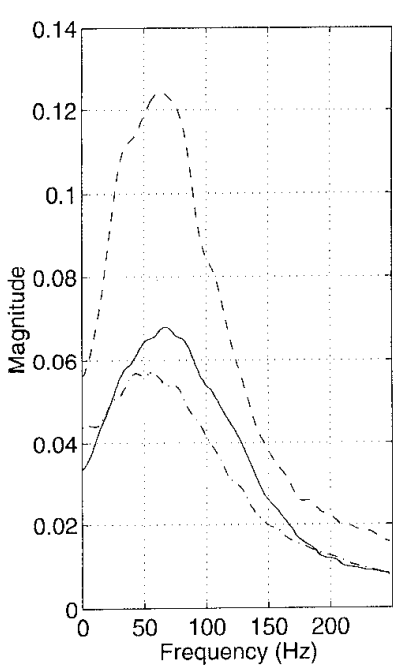

(a)

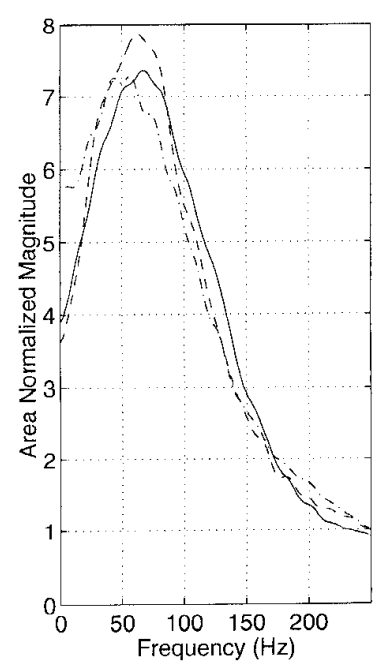

(b)
Fig. 10. Mean spectra from three thumb motions [abduction (-) (60 trials), extension (- ) (53 trials), and flexion (-.) (54 trials)] show some differences in the frequency distribution of myoelectric energy reaching the skin surface.

The 4 numbers representing each of the 6 consecutive 40 $\mathrm{ms}$ windows are inputs to a $24-6-2$ multilayer perceptron. The network outputs are: 1) [1 0$]=$ chuck grasp; 2) [0 1] = key grasp; 3) $[00]=$ no grasp; and 4) $[11]=$ ambiguous grasp. In the first two cases, the UMDH executes the grasp selection. In the latter two cases, the UMDH does nothing.

The current system response time to an operator's forming a particular grasp is just under $0.6 \mathrm{~s}$ from the operator's motion start to the UMDH's motion start. While adequate for a concept demonstration, this is too slow for an operational system. The algorithm requires $240 \mathrm{~ms}$ of myoelectric data, 
of which approximately $180 \mathrm{~ms}$ are captured after motion start. The remaining delay (approximately $0.4 \mathrm{~s}$ ) is primarily computation. Our current demonstration relies on a $25 \mathrm{MHz}$ 80386 SX (the Radix PC) for both computing FFT's and classifying the spectral signatures. Faster, specialized computing hardware such as the TMS320C3x DSP series can reduce the computation time to a few ms. This translates into a system response time under $0.2 \mathrm{~s}$, which is within acceptable realtime bounds.

Currently, operators train the system every time they don the three electrodes required. No rigorous tests have been done yet for robustness of a training session to electrode repositioning. The operator needs thirty to fifty training grasps, approximately half key and half chuck, to train the Hamming spectrogram system. If a false trigger occurs during training, the system matches that with a "no grasp" output for training to respond properly with no action to false triggers. The number of these depends on the trigger threshold: lower thresholds result in more false triggers and hence require more training signatures. Higher thresholds increase risk of missed commands, particularly where command motion velocity varies a lot. Triggering is not as simple as it appears: we tested five triggering approaches before adopting the energy change algorithm now in the MTDS [44]. Ironically, a very clean myoelectric signal measurement makes triggering more difficult than for one corrupted by large motion artifacts.

The number of training grasps must take into account the fact that the training data is split into a training subgroup $(75 \%$ of training signatures) and a training test group (the remaining $25 \%$ ). Off-line and real-time experience with applying multilayer perceptrons to myoelectric data showed that data other than the training input set is needed to determine the training quality. Consequently, we base the error computation used to decide whether to quit or continue training after each iteration on the network's performance on the training test group as well as the training subgroup. The traditional least-squared error criteria computed strictly from the training data being presented to the network results in an over-trained network that performs poorly on data not in the training set. Using the actual selection error from a combination of the training data (the training subgroup) and other data (the training test group) is most effective.

Around 300 training iterations are generally sufficient to find a minimum multilayer-perceptron selection error on the entire training set. This takes only a few minutes and results in an operational system which performs up to $95 \%$ correct grasp selection without taking triggering issues into account. Incorporating the "no grasp" option to take triggering problems into account causes the overall performance to drop to near $90 \%$. A detailed examination of a typical run (Table I) suggests why.

The $27 \%$ training error seems unacceptably high; however, the network's operational performance was far better than that, at $90 \%$ including the triggering complications $(92.5 \%$ without). The problem in this run was too few false trigger or "no grasp" training options. These are difficult to artificially generate, and there is a lot of variation in their myoelectric
TABLE I

Statistics for a SPECIFiC Teleoperation Session Using the Hamming Periodogram Algorithm

\begin{tabular}{|c|c|c|}
\hline \multirow[t]{5}{*}{ Training } & \multirow[t]{3}{*}{ Total training grasps } & 20 Chuck \\
\hline & & $20 \mathrm{Key}$ \\
\hline & & 8 False Triggers \\
\hline & Minimum Training Error & $27 \%$ \\
\hline & Training iterations & 304 \\
\hline \multirow[t]{4}{*}{ Performance } & Total grasp trials & 138 \\
\hline & \multirow[t]{2}{*}{ Error } & $\begin{array}{l}7 \% \text { wrong grasp } \\
\text { choice }\end{array}$ \\
\hline & & $3 \%$ false triggers \\
\hline & Correct & $90 \%$ \\
\hline
\end{tabular}

signature's characteristics, as they may be due to background noise, operator's arm motion, or residual muscle tension.

Fig. 11 shows the consolidated results from the specific run detailed above in terms of means and standard deviations of the features [(a) chuck and (d) key]. Recall that features 1-4 represent the first $40 \mathrm{~ms}$ window's $78-265 \mathrm{~Hz}$ spectrum, features 5-8 the second $40 \mathrm{~ms}$ window, and so on. Figs. 4-11(b) and (c) show the separation distances (solid lines) between the chuck and key superimposed on the key and chuck feature standard deviations (dashed lines).

These separation distances suggest that the overall feature magnitude in each window was the most important factor in classification. Fig. 11(e) shows the overall feature magnitude (solid line) in each window for chuck $(o)$ and key $(+)$ test cases. We attribute the difference in magnitude to several physiological factors. First, the thumb has farther to travel in abducting toward opposition of the index finger in the chuck grip than it does in flexing toward the side of the index finger in the key grip. Second, in the chuck grip the thumb is moving against (stretching) palm fascia and carpometacarpal joint connective tissue. The thumb is moving with palm fascia in the range of motion required by the key grasp. Finally, the FPL's smaller companion Flexor Pollicis Brevis (FPB), located in the hand itself, far from the electrode, assumes a progressively greater portion of the force application as the thumb comes into contact with the index finger.

Fig. 11(e) also shows the respective mean frequencies computed over the 78-265 $\mathrm{Hz}$ range only (dotted lines); these are virtually identical for chuck and key grasps. This is not surprising, since limiting the mean frequency calculation to this range makes it more of a measure of concavity or convexity than anything else.

Fig. 11(f) shows a version of the chuck (solid line) and key (dash-dot line) features normalized by their respective areas under the spectrum in each window. Here, it is clear that spectrum shape is a distant second to magnitude in importance. The slope of the spectrum's roll-off as frequency increases is less for the key grasp than for the chuck. This is probably due to less EPB/APL activity and greater FPL activity in the key than in the chuck, combined with greater low-pass tissue filtering on the FPL side. However, this slope difference is very small compared to the magnitude. This explains why performance did not improve with additional hidden layer 


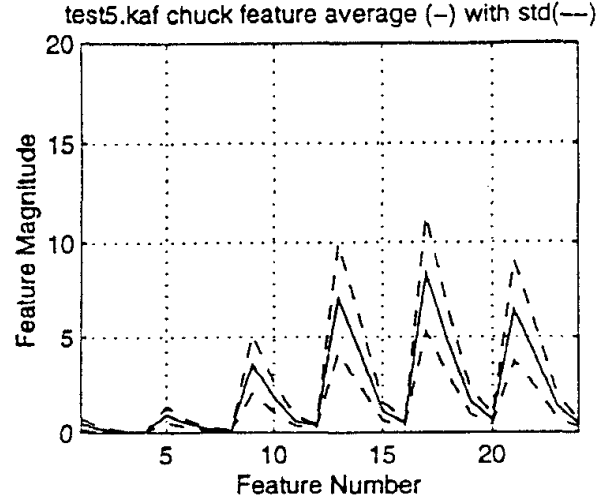

(a)

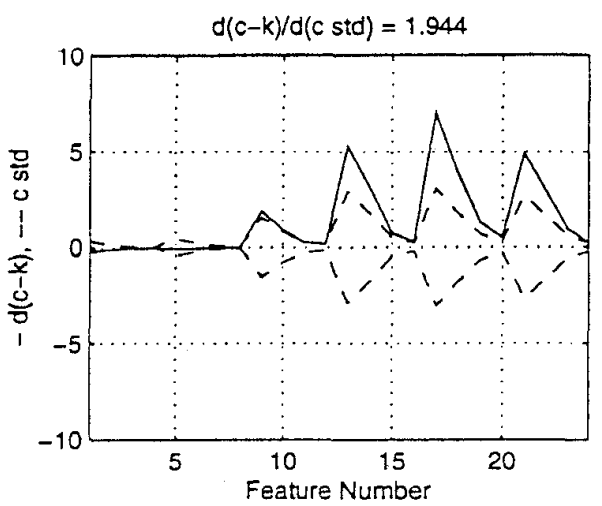

(c)

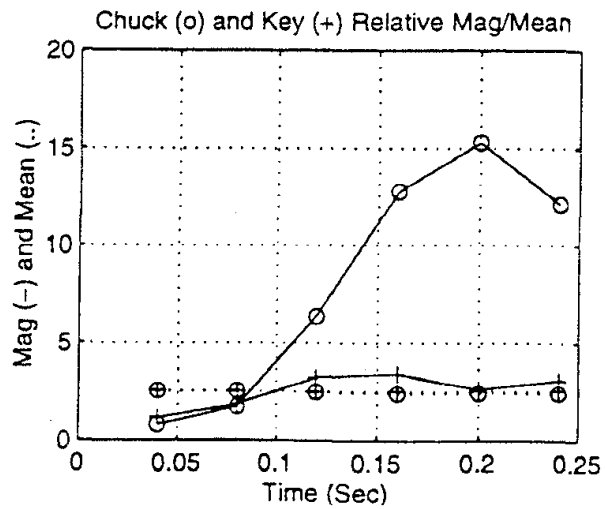

(e)

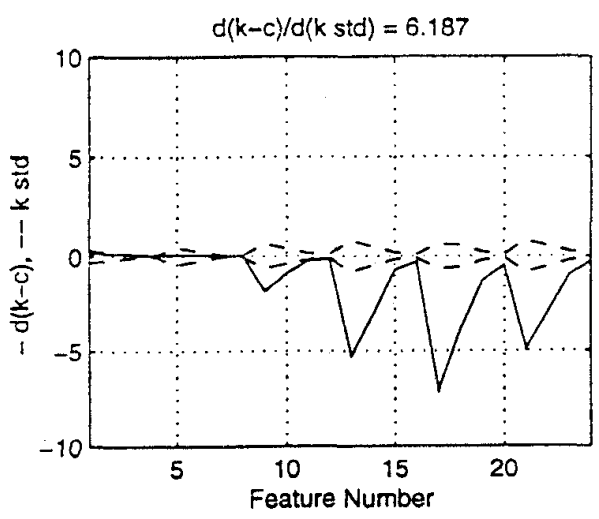

(b)

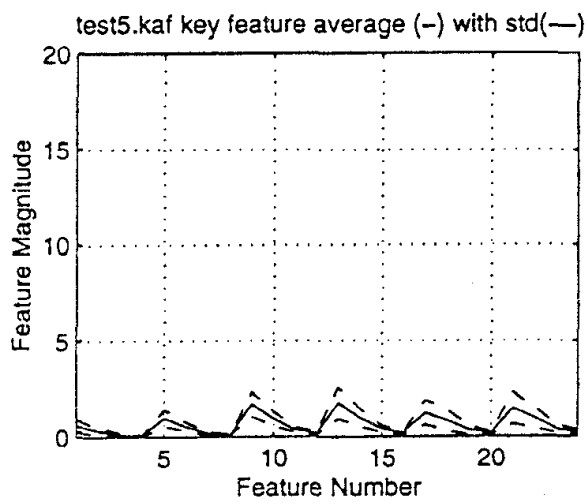

(d)

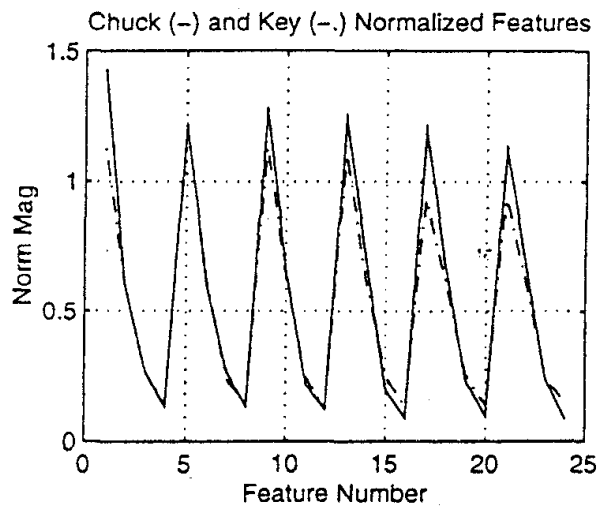

(f)

Fig. 11. Performance analysis for a sample run of the Hamming spectrogram algorithm. (a) and (d) show average features (-) and their standard deviations (STD's) (- -), (b) and (c) superimpose feature separation distances (-) over STD's (- -), (e) superimposes chuck (o) and key (+) grasp magnitude (-) and mean frequencies (..). (f) superimposes normalized chuck $(-)$ and key (-) features. Spectral detail is lost in the spectrogram algorithm, prompting us toward the STTT approach

units: the network emphasizes magnitude versus time in its classification.

The two signature magnitudes are very close in the first two windows, suggesting that motion artifact swamps out the myoelectric activity differences here, the trigger threshold was too low, or too much pre-trigger data was incorporated. Starting the feature computation later might reduce overhead without affecting performance.

While a $90 \%$ classification is respectable, this reliance on magnitude raises concerns that the approach will not expand to discriminating more than two motions, particularly given the feature's large standard deviations [Fig. 11(a) and (d)]. The algorithm's difficulty in distinguishing the low magnitude key grasp from the false triggers ( $29 \%$ of the errors were mix-ups between false triggers and key grasps) tends to confirm this suspicion.

Another concern is the amount of digital or analog computation (and hence hardware, weight, and power supply) required for this algorithm compared to one that computes just the upper spectrum energy at 6 points in time-such an algorithm might perform nearly as well as the six window Hamming spectrogram algorithm implemented here with less hardware. 
Using the upper spectrum slope in addition to magnitude at the six points would give the same separation.

\section{CONCLUSIONS AND FUTURE WORK}

We recognize that a $90 \%$ success rate for two-way grasp discrimination and $85 \%$ success rate for three-way thumb motion discrimination is not adequate for a teleoperation application. Note, however, that we achieved these results using only one myoelectric channel. We expect that the use of additional channels will improve accuracy.

We are continuing work to improve both the success rate and the scope of our controller. Our analysis of the myoelectric signature has led us to Thomson's MWM to estimate the myoelectric spectrum [57], [58]. We are now working on a realtime implementation of the STTT for calculating signal features, since there is greater separation between motionspecific Thomson spectra than the periodograms used in the demonstration described above. To support this, we are upgrading to the MDCS and MTDS to higher sample rates and greater computational throughput to achieve greater resolution on each myoelectric channel as well as multiple channel capability.

We can implement the STTT on the same digital signal processing (DSP) hardware package that we would use for an embedded operational (as opposed to laboratory) version of the Hamming spectrogram approach tested here. For each $64 \mathrm{~ms}$ window, the crude STTT estimate using $N W=$ 2 requires 4 of the length 64 FFT solutions, four times that of the Hamming spectrogram, plus 256 adds and 256 multiplies not required for the spectrogram. The STTT feature computation, however, does not require as many windows as the Hamming spectrogram, since it has less variance in longer windows than the spectrogram. We also make use of the lower spectrum $(0-75 \mathrm{~Hz})$ instead of just the $75-250 \mathrm{~Hz}$ range, since the STTT has much less bias and variance than the spectrogram. For example, the motion could be covered with three nonoverlapping $64 \mathrm{~ms}$ windows. Experiments with the Texas Instruments' TMS320C31 DSP chip shows it is an excellent candidate that can handle these operations in realtime, even at substantially faster sample rates to increase resolution [68].

Even if additional work raises our success rate appreciably above the $90 \%$ we have already obtained, we still have much work to do to make this an operational teleoperation approach. For example, grasps done while using unrelated muscles in the arm to support the arm or wrist must also be studied, to determine if these motions can be separated from the desired hand motions. We must also broaden our analysis to more than the two subjects used here. The frequency signatures will vary from individual to individual as well as change somewhat from day to day for a given individual. A method to differentiate hand motion from myoelectric signals must be applicable to any individual on any day and be insensitive to imprecise electrode positioning. This robustness must be achieved without excessive repetitive user training.

Our work represents the critical first steps toward teleoperation of multiple functions of a robotic hand using myoelectric signals. In particular, this is the first intuitive myoelectric teleoperation in which a complex robot hand reproduces the motions of the operator's hand in realtime. We have achieved this without bulky or restrictive exoskeletons or nonintuitive hand controllers. We have yet to attain the accuracy level of these more traditional controllers with a myoelectric system, but our work demonstrates the potential for a myoelectric competitor. This work also begins the process of defining the upper bounds of myoelectric multifunction hand control for the prosthetics community.

\section{ACKNOWLEDGMENT}

NASA/Johnson Space Center (JSC) provided research equipment, including the EXOS ${ }^{\mathrm{TM}}$ exoskeleton, Utah/MIT Dextrous Hand, and myoelectric measurement equipment. We would like to acknowledge the contributions of J. Clark, D. Johnson, and R. Massey of Rice University; J. Erickson, C. Hess, and L. Li of NASA/JSC; R. Davis of Lockheed; R. Scott, B. Hudgins, and K. Englehart of the University of New Brunswick; and C. Bordelon of Baylor College of Medicine. Our realtime embedded FFT routine is by Ron Mayer and uses and a stable trig generator by $\mathrm{O}$. Buneman.

\section{REFERENCES}

[1] W. Fisher and C. Price, Space Station Freedom External Maintenance Task Team. NASA/JSC Pub., 1990.

[2] S. Jacobsen et al., "Design of the Utah/MIT dextrous hand," in Proc. 1986 IEEE Int. Conf. Robotics and Automation, San Francisco, Apr $7-10,1986$.

[3] K. Farry and I. Walker, "Myoelectric teleoperation of a multifingered artificial hand," in Proc. 7 th World Cong. of the Int. Soc. of Pros. and Orth., Chicago, June 28-July 3, 1992, p. 186.

[4] "Myoelectric teleoperation of a complex robotic hand," in Proc 1993 IEEE Int. Conf. on Robotics and Automation, Atlanta, May 3-5, 1993, pp. 502-509.

[5] M. Nader, "The substitution of missing hands with myoelectric prostheses," Clinical Orthopaedics and Related Research, no. 258, pp. 9-17, Sept. 1990.

[6] C. Almstrom et al., "Experience with Swedish multifunctional prosthetic hands controlled by pattern recognition of multiple myoelectric signals," in Int. Orthopaedics. Berlin: Springer-Verlag, 1981, vol. 5, pp. 15-21.

[7] L. Leifer, "Prostheses," in 1987 Enc. of Artificial Intelligence, S. C. Shapiro, Ed. Wiley Interscience Pub.: John Wiley \& Sons, 1987, vol. 2, pp. 797-805.

[8] Limbs of Love, "An American initiative: The next generation of myoelectric prostheses," NASA/JSC Workshop Report, Res. Triangle Inst., 1991.

[9] M. Mason and J. Salisbury, Robot Hands and the Mechanics of Manipulation. Cambridge, MA: MIT Press, 1985.

[10] M. Cutkosky, Robotic Grasping and Fine Manipulation. Boston: Kluwer, 1985.

[11] J. Salisbury and J. Craig, "Articulated hands: Force control and kine matic issues," Int. J. Robot. Res., vol. 1, pp. 4-17, 1982.

[12] S. Narasimham, "Dextrous robotic hands: Kinematics and control," M.S Thesis, Dept. Elec. Eng. Comp. Sci., Massachustees, Inst. Technology, Cambridge, Jan. 1988.

[13] T. Speeter, "Control of the Utah/MIT hand: Hardware and software hierarchy," J. Robot. Sys., vol. 7, no. 5, pp. 759-790, May 1990.

[14] H. Kay and M. Rakic, "Specifications for electro-mechanical hands," in Proc. 4th Int. Symp. Ext. Control of Human Extremities, Dubrovnik, Aug. 28-Sept. 2, 1972

[15] M. Spinner, Kaplan's Functional and Surgical Anatomy of the Hand, 3rd ed. Philadelphia: JB Lippincott, 1984, pp. 9-10.

[16] R. Todd and J. Nightingale, "Adaptive prehension control for a prosthetic hand," in 3rd Int. Symp. Ext. Control of Human Extremities, Dubrovnik, Aug. 25-30, 1969, pp. 171-183.

[17] A. Georgopoulos et al, "Cognitive neurophysiology of the motor cortex," Science, vol. 260, pp. 47-52, 1993. 
[18] I. Kato and K. Okazaki, "Electro-pneumatically controlled artificial hand," Bul. of Science and Eng. Research Lab., Waseda Univ., no. 44, pp. 25-34, 1969.

[19] G. Hagg and K. Spets, "SVEN_Project I-Electrically controlled hand prosthesis-Final report," Res. Inst. of the Swedish National Defence. Dept. 2, S-104 50, Stockholm 80, FOA 2 Rep. A 2575-H5, 1973.

[20] G. Hagg and K. Oberg, "Adaptive EMG-controlled hand prosthesis for wrist disarticulated patients," Ean-Holmgren Orthopaedic Co., Bergsbrunnagatan 1, S-753 23 Uppsala, Sweden, pp. 441-449, 1975.

[21] Y. Lozach et al., "On the evaluation of a multifunctional prosthesis," in Proc. 7th World Cong. of the Int. Soc: of Pros. and Orth., Chicago, June 28-July 3, 1992, p. 185.

[22] N. Hogan, "A review of the methods of processing EMG for use as a proportional control signal," Biomed. Eng., pp. 81-86, Mar. 1976.

[23] Berne et al., Physiology, 2d ed. St. Louis: C.V. Mosby, 1988, pp. 315-348.

[24] P. Parker et al., "Signal processing for the multistate myoelectric channel," in Proc. IEEE, vol. 65, no. 5, pp. 662-674, May 1977

[25] R. Reiter, "Eine neue elecktrokunsthand," Grenzgebiete der Medizin, vol. 4 , p. $183,1948$.

[26] R. Mann, "Cybernetic limb prosthesis: The ALZA distinguished lecture," Ann. Biomed. Eng., vol. 9, pp. 1-43, 1991.

[27] J. Kreifeldt and S. Yao, "A signal-to-noise investigation of nonlinear electromyographic processors," IEEE Trans. Biomed. Eng., vol. BME21, no. 4, pp. 298-308, July 1974.

[28] A. Bottomley, "Progress with the British myoelectric hand," in Proc: 2nd Int. Symp. Ext. Control of Human Extremities, Dubrovnik, 1966.

[29] D. Childress, "An approach to powered grasp," in Proc. 4th Int. Sym. on Ext. Control of Human Extremities, Dubrovnik. Aug. 28-Sept. 2, 1972.

[30] J. Kreifeldt, "Signal versus noise characteristics of filtered EMG used as a control source," IEEE Trans. Biomed. Eng., vol. BME-18, no. 1, pp. 16-22, Jan. 1971.

[31] N. Hogan and R. Mann, "Myoelectric signal processing: Optimal estimation applied to electromyography," IEEE Trans. Biomed. Eng., vol. BME-27, no. 7, pp. 382-410, July 1980.

[32] S. Jacobsen et al., "Development of the Utah artificial arm," IEEE Trans. Biomed. Eng., vol. BME-29, no. 4, pp. 249-269, Apr. 1982.

[33] S. Meek, "An adaptive myoelectric filter," M.S. Thesis, Dept. Mech. and Industrial Eng., Univ. of Utah, Salt Lake City, June 1979.

[34] R. Wirta and D. Taylor, "Development of a multiple-axis myoelectrically controlled prosthetic arm," in Proc. 3rd Int. Sym. on Ext. Control of Human Extremities, Dubrovnik, Aug. 25-30, 1969

[35] C. Almstrom, "Myoelectric control of multifunctional hand prosthe ses--Contributions to the pattern recognition approach, to signal acquisition, and to clinical evaluation," Tech. Rep. 79, School of Elec. Eng., Chalmers Univ. of Tech., Göteborg, Sweden, 1977.

[36] P. Herberts et al., "Hand prosthesis control via myoelectric patterns," Acta Orthopaedia Scandinavia, vol. 44, pp. 389-409, 1973.

[37] S. Jacobsen, "Control systems for artificial arms," Ph.D. Dissertation, Dept. Mech. Eng., Massachusetts Inst. Technology, Cambridge, , Jan. 1973.

[38\} R. Jerard, "Application of a unified theory for simultaneous multiple axis artificial arm control," Ph.D. dissertation, Dept. Mech. and Ind. Eng., Univ. of Utah, Salt Lake City, Dec. 1976.

[39] J. Basmajian and C. DeLuca, Muscles Alive, 5th ed. Baltimore: Williams and Wilkins, 1985.

[40] L. Lindstrom and R. Magnusson, "Interpretation of myoelectric power spectra: A model and its applications," Proc. IEEE, vol. 65, no. 5, pp. 653-662, May 1977.

[41] E. Park and S. Meek, "Fatigue compensation of the electromyographic signal for prosthetic control and force estimation," IEEE Trans. Biomed. Eng., vol. 40, no. 10, pp. 1019-1023, Oct. 1993.

[42] C. DeLuca, "Physiology and mathematics of myoelectric signals," IEEE Trans. Biomed. Eng., vol. BME-26, no. 6, pp. 313-325, June 1979.

[43] B. Hannaford and S. Lehman, "Short-time Fourier analysis of the electromyogram: Fast movements and constant contraction," IEEE Trans. Biomed. Eng., vol. BME-33, no. 12, pp. 1173-1181, Dec. 1986.

[44] K. Farry, "Issues in myoelectric teleoperation of complex artificial hands," Ph.D dissertation, Elec. and Computer Eng., Rice University, Houston. TX, Nov. 1994.

[45] D. Graupe and W. Cline, "Functional separation of EMG signals via ARMA identification methods for prosthesis control purposes," IEEE Trans. Syst., Man, Cybern., vol. SMC-5, no. 2, pp. 252-259, Mar. 1975.

[46] D. Graupe et al., "A microprocessor system for multifunctional control of upper-limb prostheses via myoelectric signal identification." IEEE Trans. Automat. Contr. vol. AC-23, no. 4, pp. 538-544, Aug. 1978.

[47] D. Graupe, "Multifunctional prosthesis and orthosis control via microcomputer identification of temporal pattern differences in single site myoelectric signals," J. Biomed. Eng., vol. 4, pp. 17-22, 1982.
[48] G. Saridis and T. Gootee, "EMG pattern analysis and classification for a prosthetic arm," IEEE Trans. Biomed. Eng., vol. BME-29, no. 6, pp. 403-412, June 1982

[49] P. Doerschuk et al., "Upper extremity limb function discrimination using EMG signal analysis," IEEE Trans. Biomed. Eng., vol. BME-30, no. 1, pp. 18-29. Jan. 1983.

[50] M. Kelly et al., "The application of neural networks to myoelectric signal analysis: A preliminary study," IEEE Trans. Biomed. Eng., vol BME-37, no. 3, pp. 221-230, Mar. 1990.

[51] B. Hudgins et al., "A new strategy for multifunction myoelectric control," IEEE Trans. Biomed. Eng., vol. BME-40, no. 1, pp. 82-94, Jan. 1993.

[52] T. Parks and C. Burrus, Digital Filter Design. New York: Wiley, 1987.

[53] A. Oppenheim et al., Signals and Systems. Englewood Cliffs, NJ: Prentice-Hall, 1987.

[54] D. Thomson, "Spectrum estimation and harmonic analysis," Proc. IEEE vol. 70, no. 9, pp. 1055-1096, Sept. 1982.

[55] J. Park et al., "Multitaper spectral analysis of high-frequency seismograms," J. Geophys. Res., vol. 92, no. B12, pp. 12675-12684, Nov. 1987.

[56] D. Slepian and H. O. Pollak, "Prolate spheroidal wave functions, Fourier analysis and uncertainty-I," Bell Systems Tech. J., vol. 40, pp. 43-64, 1961.

[57] K. Farry et al., "Myoelectric spectrum estimation using Thomson's multiple window method: Single signal stationary analysis," submitted to IEEE Trans. Biomed. Eng., June 1995.

[58] __. "Myoelectric spectrum estimation using Thomson's multiple window method: Time-frequency analysis," submitted to IEEE Trans. Biomed. Eng., Aug. 1995.

[59] Exos, Inc., Dextrous Hand Master Manual, 1991 Tech. Manual, Exos, Inc., 8 Blanchard Road, Burlington, MA.

[60] A. Wright and M. Stanisic, "Kinematic mapping between the EXOS handmaster exoskeleton and the Utah/MIT dextrous hand," in Proc. IEEE Int. Conf. Sys. Eng., Pittsburgh, PA, Aug. 9-11, 1990, pp. 101-104

[61] Grass Instruments, Inc., Model 12 Neurodata Acquisition System for EEG, Evoked Potentials, and EMG, 1986 Tech. Manual, 101 Old Colony Ave., Quincy, MA 02269-0516.

[62] The MathWorks, Inc., MATLAB, Version 4.X, 24 Prime Park Way, Natick, MA 01760

[63] S. Olmstead and F. Watkins, OWL Applications Development Package, Version 1.0, for IBM PC Compatibles, 1988 Tech. Manual, Olmstead and Watkins, 2411 East Valley Parkway, Suite 294, P.O. Box 3751, Escondido, CA 92025.

[64] S. M. Kay, Modern Spectral Estimation: Theory and Application. Englewood Cliffs, NJ: Prentice-Hall, 1988.

[65] A. Drosopoulis and S. Haykin, in "Adaptive radar estimation with Thomson's multiple window method," Adaptive Radar Estimation Haykin and Steinhardt, Eds. New york: Wiley, 1992, pp. 381-461.

[66] E. Clancy and N. Hogan, "Single site electromyographic amplitude estimation," IEEE Trans. Biomed. Eng., vol. 41, no. 2, pp. 159-167, Feb. 1994.

[67] K. Farry et al, "Functional separation of myoelectric signals using Thomson's multiple window method," in Myoelectric Control '95, University of New Brunswick, Fredericton, New Brunswick, Canada, Aug. 14-17, 1995, pp. 57-66.

[68] electrics," Myoelectric Control '93. University of New Brunswick, Fredericton, New Brunswick, Canada, Aug. 16-20, 1993, pp. 115-122.

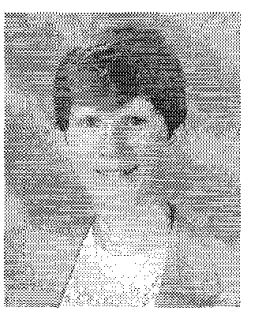

Kristin A. Farry ( $\left.\mathrm{S}^{\prime} 93-\mathrm{M}^{\prime} 94\right)$ received the B.S degree in aerospace engineering. from the University of Virginia, Charlottesville, in 1980, the M.S.E. degree from Princeton University, Princeton, $\mathrm{NJ}$, in 1988, and the Ph.D. in electrical engineering from Rice University, Houston, TX, in 1995.

From 1980 to 1982, she was with Princeton University's Flight Research Laboratory, developing a "fly-by-light" (airborne fiberoptics) system. From 1982 to 1986, she was a U.S. Air Force officer assigned to the Flight Dynamics Laboratory, working on aircraft (X-29) and robotics control systems. From 1986 to 1990 she worked for Rockwell International on space station, national aerospace plane (NASP), and shuttle Mission Control (Remote Manipulator System). Since 1994, she has been a National Research Council Research Associate at NASA's Johnson Space Center. Her research interests include space robotics, myoelectric control, upper limb prosthetics, and aerospace vehicles.

Dr. Farry is a member of Tau Beta Pi and Sigma Gamma Tau. 


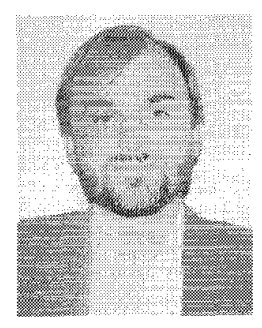

Ian D. Walker (S'84-M'89) received the B.Sc degree in mathematics from the University of Hull England, in 1983, and the M.S. and Ph.D. degrees, both in electrical engineering, from the University of Texas at Austin, in 1985 and 1989, respectively. Since 1989, he has been with the Department of Electrical and Computer Engineering, Rice University, Houston, TX, where he is currently an Associate Professor. His research interests are in the areas of robotics and control, particularly robotic hands and grasping; fault tolerant robot systems; and kinematically redundant robots.

Dr. Wakker is a member Beta Alpha Phi, Eta Kappa Nu, Phi Kappa Phi, and Tau Beta Pi.

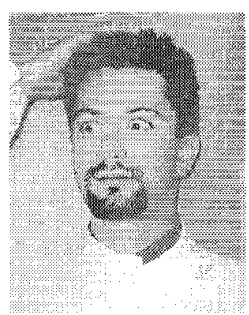

Richard G. Baraniuk (M'93) received the B.Sc. degree in 1987 from the University of Manitoba, Winnipeg, Manitoba, Canada, the M.Sc. degree in 1988 from the University of Wisconsin-Madison, and the Ph.D. degree in 1992 from the University of Illinois, Urbana-Champaign, all in electrical engineering.

In 1986 , he was a research engineer with Omron Tateisi Electronics in Kyoto, Japan. After spending 1992 and 1993 with the Signal Processing Laboratory of Ecole Normale Superieure, Lyon, France, he joined Rice University, Houston, TX, as an Assistant Professor of Electrical and Computer Engineering. His research interests lie in the area of signal and image processing and include wavelet theory and time-frequency analysis.

Dr. Baraniuk received a NATO postdoctoral fellowship from NSERC in 1992, a National Young Investigator award from the National Science Foundation in 1994, and a Young Investigator award from the Office of Naval Research in 1995. 